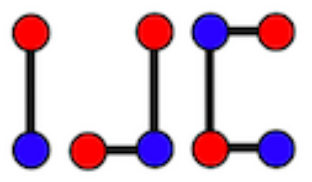

\title{
Edge irregular reflexive labeling on sun graph and corona of cycle and null graph with two vertices
}

Irfan Setiawan, Diari Indriati

Department of Mathematics, Faculty of Mathematics and Natural Sciences, Universitas Sebelas Maret sunirfan74@gmail.com,diari_indri@yahoo.co.id

\begin{abstract}
Let $G(V, E)$ be a simple and connected graph which set of vertices is $V$ and set of edges is $E$. Irregular reflexive $k$-labeling $f$ on $G(V, E)$ is assignment that carries the numbers of integer to elements of graph, such that the positive integer $\left\{1,2,3, \ldots, k_{e}\right\}$ assignment to edges of graph and the even positive integer $\left\{0,2,4, \ldots, 2 k_{v}\right\}$ assignment to vertices of graph. Then, we called as edge irregular reflexive $k$-labelling if every edges has different weight with $k=\max \left\{k_{e}, 2 k_{v}\right\}$. Besides that, there is definition of reflexive edge strength of $G(V, E)$ denoted as $\operatorname{res}(G)$, that is a minimum $k$ that using for labeling $f$ on $G(V, E)$. This paper will discuss about edge irregular reflexive $k$ labeling for sun graph and corona of cycle and null graph, denoted by $C_{n} \odot N_{2}$ and make sure about their reflexive edge strengths.
\end{abstract}

Keywords: Edge irregular reflexive labeling, reflexive edge strength, sun graph, corona of cycle and null graph Mathematics Subject Classification: 05C78

DOI: $10.19184 /$ ijc.2021.5.1.5

Received: 24 August 2020, Revised: 24 January 2021， Accepted: 29 April 2021. 


\section{Introduction}

All graph discuss on this paper are simple, connected, and undirected. First, let simplified $G(V, E)$ become $G$, vertex of graph denoted as $v$, and edge of graph denoted as $e$ for easily on investigation. By definition from Wallis and Marr [10], graph labeling is a map that carries graph elements to numbers, usually to positive integer. The kinds of graph labeling divided as vertexlabelings, edge-labelings, and total labeling (vertex and edge-labelings). Based on survey that launch by Galian [5], there are many kinds of graph labelling. One of them is irregular total $k$ labeling.

According to Bača et al. [8], irregular total $k$-labeling divided be two kinds, that is edge irregular total $k$-labeling and vertex irregular total $k$-labeling. In 2017, Ryan et al. [6] has introduced new concept about irregular total $k$-labeling, that is vertex irregular reflexive total $k$-labeling and edge irregular reflexive total $k$-labeling. We call edge irregular reflexive total $k$-labeling if labeling $f$ on $G$ caries the positive integers 1 until $k_{e}$ to edges of graph and caries the even positive integers 2 until $2 k_{v}$ to vertices of graph with $k=\max \left\{k_{e}, 2 k_{v}\right\}$. The other spesifications edge irregular reflexive total $k$-labeling is all of edges have different weight. The mapping positive integers to edge $x y$ of graph denoted as $f(x y)$ and the mapping even positive integers to vertex $x$ of graph denoted as $f(x)$. Moreover, the weight edge $x y$ of graph denoted as $w t(x y)$, where $w t(x y)=$ $f(x)+f(x y)+f(y)$.

This paper also make investigate about reflexive edge strength of $G$, denoted as $\operatorname{res}(G)$. To determine a lower bound of $\operatorname{res}(G)$ Ryan [6] gave a Lemma 1.1 for all graph $G$,

\section{Lemma 1.1.}

$$
\operatorname{res}(G) \geq\left\{\begin{array}{lll}
\left\lceil\frac{|E(G)|}{3}\right\rceil, & \text { if }|E(G)| \neq \equiv 2,3 & (\bmod 6), \\
\left\lceil\frac{|E(G)|}{3}\right\rceil+1, & \text { if }|E(G)| \equiv 2,3 & (\bmod 6) .
\end{array}\right.
$$

Some of $\operatorname{res}(G)$ has been determined, such as prisms graph $D_{n}$ [2], cycle $C_{n}$ [7], wheels graph $W_{n}$ [2], corona of path and other graph, $P_{n} \odot K_{1}$ and $P_{n} \odot P_{2}$ [1], and other. This paper will investigate about sun graph and corona of cycle and null graph $N_{2}$.

\section{The sun graph}

Based on definition of sun graph by Wallis and Marr [10], an $n$-sun is a cycle $C_{n}$ with an edge terminating in a vertex of degree 1 attached to each vertex and by Boulet [9] denoted as $\operatorname{Sun}_{n}$. Then we will denote vertices in the cycle as $x_{i}$ and pendant vertices denoted as $y_{i}$. So set of vertices $V\left(\operatorname{sun}_{n}\right)=\left\{x_{i}, y_{i}: 1 \leq i \leq n\right\}$, consequently set of edges $E\left(\operatorname{sun}_{n}\right)=\left\{x_{i} x_{i+1}, x_{i} y_{i}\right.$ : $1 \leq i \leq n\}$. As a result $\operatorname{sun}_{n}$ has $2 n$ edges and vertices. $\operatorname{Res}(G)$ of $\operatorname{sun}_{n}$ can be obtained by Theorem 2.1.

Theorem 2.1. For sun $_{n}$ with $n \geq 3$,

$$
\operatorname{res}\left(\operatorname{sun}_{n}\right) \doteq \begin{cases}3, & \text { if } n \doteq 3 \\ 2\left\lceil\frac{n}{3}\right\rceil, & \text { if } n>3\end{cases}
$$


Proof. First, we prove the lower bound of res( $\left.\operatorname{sun}_{n}\right)$. Since $|E|$ of $\operatorname{sun}_{n}$ is $2 n$, then by Lemma 1.1 we get

$$
\operatorname{res}\left(\operatorname{sun}_{n}\right) \geq\left\{\begin{array}{lll}
\left\lceil\frac{2 n}{3}\right\rceil, & \text { if } 2 n \neq 2,3 & (\bmod 6), \\
\left\lceil\frac{2 n}{3}\right\rceil+1, & \text { if } 2 n \equiv 2,3 & (\bmod 6) .
\end{array}\right.
$$

Let us prove the condition if $n \doteq 3$,

Graph $\operatorname{sun}_{3}$ has 6 vertices and 6 edges. By (1) we get the lower bound of $\operatorname{res}\left(\operatorname{sun}_{3}\right)=2$. Let us assume $\operatorname{res}\left(\operatorname{sun}_{3}\right)=2$, we get maximum label of vertex and label of edges is 2 , consequently the possibility of vertices and edges label for 6 edges $x_{i} y_{i}(1 \leq i \leq 6)$ with the edge weight from 1 until 6 are,

$$
\begin{aligned}
& w t_{f}\left(x_{1} y_{1}\right)=f\left(x_{1}\right)+f\left(x_{1} y_{1}\right)+f\left(y_{1}\right)=0+1+0=1 \\
& w t_{f}\left(x_{2} y_{2}\right)=f\left(x_{2}\right)+f\left(x_{2} y_{2}\right)+f\left(y_{2}\right)=0+2+0=2 \\
& w t_{f}\left(x_{3} y_{3}\right)=f\left(x_{3}\right)+f\left(x_{3} y_{3}\right)+f\left(y_{3}\right)=0+1+2=3 \\
& w t_{f}\left(x_{4} y_{4}\right)=f\left(x_{4}\right)+f\left(x_{4} y_{4}\right)+f\left(y_{4}\right)=0+2+2=4 \\
& w t_{f}\left(x_{5} y_{5}\right)=f\left(x_{5}\right)+f\left(x_{5} y_{5}\right)+f\left(y_{5}\right)=2+1+2=5 \\
& w t_{f}\left(x_{6} y_{6}\right)=f\left(x_{6}\right)+f\left(x_{6} y_{6}\right)+f\left(y_{6}\right)=2+2+2=6
\end{aligned}
$$

But, its form cannot be applied. Then, let us make assume that $\operatorname{res}\left(\operatorname{sun}_{n}\right)=3$, we get $\max$ $f\left(x_{i}\right)=2$ and $\max f\left(x_{i} y_{i}\right)=3$ for $1 \leq i \leq 6$, consequently the possibility label of vertices and edges as follows,

$$
\begin{aligned}
& w t_{f}\left(x_{1} y_{1}\right)=f\left(x_{1}\right)+f\left(x_{1} y_{1}\right)+f\left(y_{1}\right)=0+1+0=1 \\
& w t_{f}\left(x_{2} y_{2}\right)=f\left(x_{2}\right)+f\left(x_{2} y_{2}\right)+f\left(y_{2}\right)=0+2+0=2 \\
& w t_{f}\left(x_{3} y_{3}\right)=f\left(x_{3}\right)+f\left(x_{3} y_{3}\right)+f\left(y_{3}\right)=0+1+2=3 \\
& w t_{f}\left(x_{4} y_{4}\right)=f\left(x_{4}\right)+f\left(x_{4} y_{4}\right)+f\left(y_{4}\right)=0+2+2=4 \\
& w t_{f}\left(x_{5} y_{5}\right)=f\left(x_{5}\right)+f\left(x_{5} y_{5}\right)+f\left(y_{5}\right)=0+3+2=5 \\
& w t_{f}\left(x_{6} y_{6}\right)=f\left(x_{6}\right)+f\left(x_{6} y_{6}\right)+f\left(y_{6}\right)=2+2+2=6
\end{aligned}
$$

Its form can be applied, so 3 is sufficient to become the lower bound of $\operatorname{res}\left(\operatorname{sun}_{3}\right)$. Then, we will prove for condition if $n>3$. There are three cases for this condition.

Firstly if $n \equiv 0(\bmod 3)$. For this case we get,

$$
\left\lceil\frac{n}{3}\right\rceil=\frac{n}{3}
$$

By (1), if $n \equiv 0(\bmod 3)$ we get $\operatorname{res}\left(\operatorname{sun}_{n}\right) \geq\left\lceil\frac{2 n}{3}\right\rceil$. Consequently by (2),

$$
\begin{aligned}
\left\lceil\frac{2 n}{3}\right\rceil & =\frac{2 n}{3} \\
& =2\left\lceil\frac{n}{3}\right\rceil .
\end{aligned}
$$

Secondly if $n \equiv 1(\bmod 3)$. For this case we get,

$$
\left\lceil\frac{n}{3}\right\rceil=\frac{n-1}{3}+1
$$

By (1), if $n \equiv 1(\bmod 3)$ we get $\operatorname{res}\left(\operatorname{sun}_{n}\right) \geq\left\lceil\frac{2 n}{3}\right\rceil+1$. Consequently by (3), 


$$
\begin{aligned}
\left\lceil\frac{2 n}{3}\right\rceil+1 & =\frac{2(n-1)}{3}+1+1 \\
& =\frac{2(n-1)}{3}+2 \\
& =2\left(\frac{n-1}{3}+1\right) \\
& =2\left\lceil\frac{n}{3}\right\rceil .
\end{aligned}
$$

Thirdly if $n \equiv 2(\bmod 3)$,

By (1), if $n \equiv 2(\bmod 3)$ we get $\operatorname{res}\left(\operatorname{sun}_{n}\right) \geq\left\lceil\frac{2 n}{3}\right\rceil$. For $n \equiv 2(\bmod 3),\left\lceil\frac{2 n}{3}\right\rceil$ has the same value as $2\left\lceil\frac{n}{3}\right\rceil$.

Next, we will prove the upper bound of $\operatorname{res}\left(\operatorname{sun}_{n}\right)$. To prove this part we construct $k$-labeling $f$ with $k=3$ if $n=3$ and $k=2\left\lceil\frac{n}{3}\right\rceil$ if $n>3$ on $\operatorname{sun}_{n}$ as follows,

For $n \geq 3$,

$$
\begin{aligned}
& f\left(x_{i}\right)= \begin{cases}0, & \text { if } i=1 \text { and } 2, \\
\frac{4 i-2}{3}, & \text { if } i=3,4, \ldots,\left\lceil\frac{n}{2}\right\rceil \text { and } i \equiv 2 \quad(\bmod 3), \\
4\left\lfloor\frac{i}{3}\right\rfloor, & \text { if } i=3,4, \ldots,\left\lceil\frac{n}{2}\right\rceil \text { and } i \neq 2 \quad(\bmod 3) .\end{cases} \\
& f\left(y_{i}\right)= \begin{cases}\frac{4 i-4}{3}, & \text { if } i=1,2, \ldots,\left\lceil\frac{n}{2}\right\rceil \text { and } i \equiv 1 \quad(\bmod 3), \\
2+4\left\lfloor\frac{i-2}{3}\right\rfloor, & \text { if } i=1,2, \ldots,\left\lceil\frac{n}{2}\right\rceil \text { and } i \neq \equiv 1 \quad(\bmod 3) .\end{cases} \\
& f\left(y_{n-(i-1)}= \begin{cases}\frac{4 i}{3}, & \text { if } i=1,2, \ldots,\left\lfloor\frac{n}{2}\right\rfloor \text { and } i \equiv 0 \quad(\bmod 3), \\
2+4\left\lfloor\frac{i}{3}\right\rfloor & \text { if } i=1,2, \ldots,\left\lfloor\frac{n}{2}\right\rfloor \text { and } i \neq \equiv 0 \quad(\bmod 3) .\end{cases} \right. \\
& f\left(x_{i} y_{i}\right)= \begin{cases}2, & \text { if } i=1 \text { and } 2 . \\
\frac{4 i-6}{3} & \text { if } i=3,4, \ldots,\left\lceil\frac{n}{2}\right\rceil \text { and } i \equiv 0 \quad(\bmod 3), \\
4\left\lfloor\frac{i}{3}\right\rfloor, & \text { if } i=3,4, \ldots,\left\lceil\frac{n}{2}\right\rceil \text { and } i \not \equiv 0 \quad(\bmod 3) .\end{cases} \\
& 1 .
\end{aligned}
$$

For $n \geq 3$ and $n \equiv 0(\bmod 6)$,

$$
f\left(x_{n-(i-1)}\right)=\left\{\begin{array}{lll}
\frac{4 i}{3}, & \text { if } i=\frac{n}{2}, \\
\frac{4 i+4}{3}, & \text { if } i=1,2, \ldots,\left(\frac{n}{2}-1\right) \text { and } i \equiv 2 \quad(\bmod 3), \\
2+4\left\lfloor\frac{i}{3}\right\rfloor, & \text { if } i=1,2, \ldots,\left(\frac{n}{2}-1\right) \text { and } i \neq 12 \quad(\bmod 3) .
\end{array}\right.
$$

For $n \geq 3$ and $n \not \equiv 0(\bmod 6)$,

$$
f\left(x_{n-(i-1)}\right)=\left\{\begin{array}{lll}
\frac{4 i+4}{3}, & \text { if } i=1,2, \ldots,\left\lfloor\frac{n}{2}\right\rfloor \text { and } i \equiv 2 \quad(\bmod 3) \\
2+4\left\lfloor\frac{i}{3}\right\rfloor, & \text { if } i=1,2, \ldots,\left\lfloor\frac{n}{2}\right\rfloor \text { and } i \neq \equiv 2 & (\bmod 3) .
\end{array}\right.
$$


For $n \geq 3$ and $n \equiv 2(\bmod 6)$,

$$
f\left(x_{n-(i-1)} y_{n-(i-1)}\right)=\left\{\begin{array}{lll}
\frac{4 i-4}{3}, & \text { if } i=\frac{n}{2}, \\
\frac{4 i+2}{3}, & \text { if } i=1,2, \ldots,\left(\frac{n}{2}-1\right) \text { and } i \equiv 1 \quad(\bmod 3), \\
4\left\lceil\frac{i}{3}\right\rceil, & \text { if } i=1,2, \ldots,\left(\frac{n}{2}-1\right) \text { and } i \neq 1 \quad(\bmod 3) .
\end{array}\right.
$$

For $n \geq 3$ and $n \equiv 4(\bmod 6)$,

$$
f\left(x_{n-(i-1)} y_{n-(i-1)}\right)=\left\{\begin{array}{lll}
\frac{4 i-2}{3}, & \text { if } i=\frac{n}{2}, \\
\frac{4 i+2}{3}, & \text { if } i=1,2, \ldots,\left(\frac{n}{2}-1\right) \text { and } i \equiv 1 \quad(\bmod 3), \\
4\left\lceil\frac{i}{3}\right\rceil, & \text { if } i=1,2, \ldots,\left(\frac{n}{2}-1\right) \text { and } i \neq 1 \quad(\bmod 3) .
\end{array}\right.
$$

For $n \geq 3$ and $n \not \equiv 2,4(\bmod 6)$,

$$
f\left(x_{n-(i-1)} y_{n-(i-1)}\right)=\left\{\begin{array}{lll}
\frac{4 i+2}{3}, & \text { if } i=1,2, \ldots,\left(\left\lfloor\frac{n}{2}\right)\right\rfloor \text { and } i \equiv 1 \quad(\bmod 3), \\
4\left\lceil\frac{i}{3}\right\rceil, & \text { if } i=1,2, \ldots,\left(\left\lfloor\frac{n}{2}\right)\right\rfloor \text { and } i \not \equiv 1 \quad(\bmod 3) .
\end{array}\right.
$$

For $n=3$,

$$
f\left(x_{i} x_{i+1}\right)=2 i-1 \text {, if } i=1,2 .
$$

For $n>3$ and $n \equiv 3(\bmod 6)$,

$$
f\left(x_{i} x_{i+1}\right)= \begin{cases}1, & \text { if } i=1 \text { and } 2, \\ \frac{4 i-5}{3}, & \text { if } i=\left\lceil\frac{n}{2}\right\rceil, \\ \frac{4 i-9}{3}, & \text { if } i=3,4, \ldots,\left(\left\lceil\frac{n}{2}\right\rceil-1\right) \text { and } i \equiv 0 \quad(\bmod 3), \\ 3+4\left\lfloor\frac{i-4}{3}\right\rfloor, & \text { if } i=3,4, \ldots,\left(\left\lceil\frac{n}{2}\right\rceil-1\right) \text { and } i \neq \equiv 0 \quad(\bmod 3) .\end{cases}
$$

For $n>3$ and $n \not \equiv 3(\bmod 6)$,

$$
f\left(x_{i} x_{i+1}\right)= \begin{cases}1, & \text { if } i=1 \text { and } 2, \\ \frac{4 i-9}{3}, & \text { if } i=3,4, \ldots,\left(\left\lceil\frac{n}{2}\right\rceil\right) \text { and } i \equiv 0 \quad(\bmod 3), \\ 3+4\left\lfloor\frac{i-4}{3}\right\rfloor, & \text { if } \left.i=3,4, \ldots,\left\lceil\frac{n}{2}\right\rceil\right) \text { and } i \neq \equiv 0 \quad(\bmod 3) .\end{cases}
$$

For $n \geq 3$ and $n \equiv 0(\bmod 6)$

$$
f\left(x_{n-(i-1)} x_{n-i}\right)=\left\{\begin{array}{lll}
\frac{4 i+1}{3}, & \text { if } i=\left(\frac{n}{2}-1\right), \\
\frac{4 i-3}{3}, & \text { if } i=1,2, \ldots,\left(\frac{n}{2}-2\right) \text { and } i \equiv 0 \quad(\bmod 3), \\
1+4\left\lfloor\frac{i}{3}\right\rfloor, & \text { if } i=1,2, \ldots,\left(\frac{n}{2}-2\right) \text { and } i \neq \equiv 0 \quad(\bmod 3) .
\end{array}\right.
$$

For $n \geq 3$ and $n \not \equiv 0(\bmod 6)$

$$
f\left(x_{n-(i-1)} x_{n-i}\right)=\left\{\begin{array}{lll}
\frac{4 i-3}{3}, & \text { if } i=1,2, \ldots,\left(\left\lfloor\frac{n}{2}\right\rfloor-1\right) \text { and } i \equiv 0 \quad(\bmod 3) \\
1+4\left\lfloor\frac{i}{3}\right\rfloor, & \text { if } i=1,2, \ldots,\left(\left\lfloor\frac{n}{2}\right\rfloor-1\right) \text { and } i \neq \equiv 0 & (\bmod 3) .
\end{array}\right.
$$


Then, by $f$-labeling we know that label of vertices is even positive integer. So the upper bound of $\operatorname{res}\left(\operatorname{sun}_{n}\right)$ has same value as the lower bound of $\operatorname{res}\left(\operatorname{sun}_{n}\right)$. The weight of edges are, For even $n$,

$$
\begin{aligned}
w t\left(x_{i} y_{i}\right) & = \begin{cases}2, & \text { if } i=1, \\
4(i-1), & \text { if } i=2,3, \ldots,\left(\frac{n}{2}+1\right) .\end{cases} \\
w t\left(x_{n-(i-1)} y_{n-(i-1)}\right) & =4 i+2, \text { if } i=1,2, \ldots,\left(\frac{n}{2}-1\right) . \\
w t\left(x_{i} x_{i+1}\right) & =4 i-3, \text { if } i=1,2, \ldots,\left\lceil\frac{n}{2}\right\rceil . \\
w t\left(x_{n-(i-1)} x_{n-i}\right) & =4 i+3, \text { if } i=1,2, \ldots,\left\lfloor\frac{n}{2}\right\rfloor . \\
w t\left(x_{1} x_{n}\right) & =3, \text { if } n \geq 3 .
\end{aligned}
$$

For odd $n$,

$$
\begin{aligned}
w t\left(x_{i} y_{i}\right) & = \begin{cases}2, & \text { if } i=1, \\
4(i-1), & \text { if } i=2,3, \ldots,\left\lceil\frac{n}{2}\right\rceil .\end{cases} \\
w t\left(x_{n-(i-1)} y_{n-(i-1)}\right) & =4 i+2, \text { if } i=1,2, \ldots,\left\lfloor\frac{n}{2}\right\rfloor . \\
w t\left(x_{i} x_{i+1}\right) & =4 i-3, \text { if } i=1,2, \ldots,\left\lceil\frac{n}{2}\right\rceil . \\
w t\left(x_{n-(i-1)} x_{n-i}\right) & =4 i+3, \text { if } i=1,2, \ldots,\left\lfloor\frac{n}{2}\right\rfloor . \\
w t\left(x_{1} x_{n}\right) & =3, \text { if } n \geq 3 .
\end{aligned}
$$

By this investigate, we can conclude that every edges has different weight, consequently $f$ is edge irregular reflexive $k$-labeling. So, the proof of $\operatorname{res}\left(\operatorname{sun}_{n}\right)$ is completed.

An illustration of edge irregular reflexive $k$-labeling on $\operatorname{sun}_{n}$ for even $n$ can be seen on Figure 1. The black color is label, red color is weight and blue color is name of vertices.

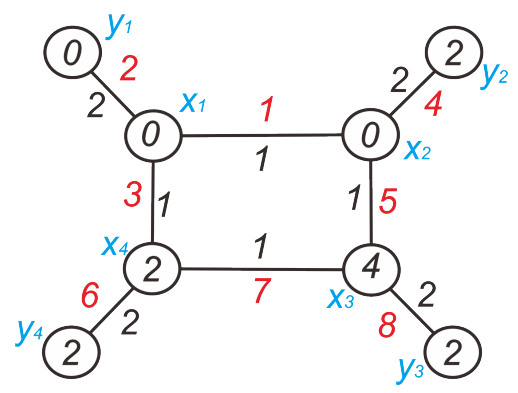

Figure 1. Edge irregular reflexive 4-labeling of $\operatorname{sun}_{4}$. 


\section{The corona of cycle and null graph with two vertices}

According Dwivedi [4], a graph $G(V, E)$ is called null graph which does not have any edges, in other words every vertex are isolated, denoted as $N_{m}$ with $m$ the number of vertices. Then, based on definition corona of two graph by Harray and Frunct [3], corona of cycle and null graph denoted as $C_{n} \odot N_{m}$ is a graph that formed by one copy of graph $C_{n}$ and $n$-copy graph of $N_{m}$ with $i-t h$ vertex from $C_{n}$ is connected to all of vertices from $i-t h$ copy of graph $N_{m}$. It is consequently that $C_{n} \odot N_{m}$ has $|E|=n(m+1)$. First, we know that $C_{n} \odot N_{1}$ same as sun graph. Therefore, in this paper we continue to discuss of $C_{n} \odot N_{2}$ for $C_{n}$ with $n \geq 3$. We will denote vertices in the cycle of $C_{n} \odot N_{2}$ as $x_{i}$ and pendant vertices denoted as $y_{i, j}$. So set of vertices $V\left(C_{n} \odot N_{2}\right)=\left\{x_{i}, y_{i, j}: 1 \leq i \leq n\right.$ and $\left.j=1,2\right\}$, consequently set of edges $E\left(C_{n} \odot N_{2}\right)=\left\{x_{i} x_{i+1}, x_{i} y_{i, j}: 1 \leq i \leq n\right.$ and $\left.j=1,2\right\}$. Then reflexive edge strength of $C_{n} \odot N_{2}$ can be found on Theorem 3.1.

Theorem 3.1. For $C_{n} \odot N_{2}$ with $n \geq 3$,

$$
\operatorname{res}\left(C_{n} \odot N_{2}\right)=2\left\lfloor\frac{n+1}{2}\right\rfloor, \text { if } n \geq 3 .
$$

Proof. First, we prove the lower bound of $\operatorname{res}\left(C_{n} \odot N_{2}\right)$. By section (3) we get $|E|$ of $C_{n} \odot N_{2}$ is $3 n$, then by Lemma 1.1 we get

$$
\operatorname{res}\left(C_{n} \odot N_{2}\right) \geq\left\{\begin{array}{lll}
\left\lceil\frac{3 n}{3}\right\rceil, & \text { if } 3 n \neq 2,3 & (\bmod 6), \\
\left\lceil\frac{3 n}{3}\right\rceil+1, & \text { if } 3 n \equiv 2,3 & (\bmod 6) .
\end{array}\right.
$$

It is equivalent with,

$$
\operatorname{res}\left(C_{n} \odot N_{2}\right) \geq\left\{\begin{array}{lll}
\mathrm{n}, & \text { if } 3 n \neq 2,3 \quad(\bmod 6), \\
\mathrm{n}+1, & \text { if } 3 n \equiv 2,3 \quad(\bmod 6) .
\end{array}\right.
$$

For $n \equiv 0(\bmod 2)$ we get,

$$
\left\lfloor\frac{n+1}{2}\right\rfloor=\frac{n}{2} .
$$

By (5), if $n \equiv 0(\bmod 2)$ we get $\operatorname{res}\left(C_{n} \odot N_{2}\right) \geq n$. Consequently by (6) we get,

$$
\begin{aligned}
n & =\frac{2 n}{2} \\
& =2\left\lfloor\frac{n+1}{2}\right\rfloor .
\end{aligned}
$$

For $n \equiv 1(\bmod 2)$ we get,

$$
\left\lfloor\frac{n+1}{2}\right\rfloor=\frac{n+1}{2} .
$$

By (5), if $n \equiv 1(\bmod 2)$ we get $\operatorname{res}\left(C_{n} \odot N_{2}\right) \geq n+1$. Consequently by (7) we get,

$$
\begin{aligned}
n+1 & =2\left(\frac{n+1}{2}\right) \\
& =2\left\lfloor\frac{n+1}{2}\right\rfloor .
\end{aligned}
$$


Next, we will prove the upper bound of $\operatorname{res}\left(C_{n} \odot N_{2}\right)$ for $n \geq 3$. To prove this part we construct $k$-labeling $f$ with $k=2\left\lfloor\frac{n+1}{2}\right\rfloor$ if $n \geq 3$ on $C_{n} \odot N_{2}$ as follows,

For $n \geq 3$,

$$
\begin{aligned}
f\left(x_{i}\right) & = \begin{cases}2(i-1), & \text { if } i=1 \text { and } 2, \\
2 i, & \text { if } i=3,4, \ldots,\left\lceil\frac{n}{2}\right\rceil .\end{cases} \\
f\left(x_{n-(i-1)}\right) & =2 i, \text { if } i=1,2, \ldots,\left\lfloor\frac{n}{2}\right\rfloor . \\
f\left(y_{i, j}\right) & = \begin{cases}0, & \text { if } i=1 \text { and } j=1,2, \\
2 i, & \text { if } i=2,3, \ldots,\left\lceil\frac{n}{2}\right\rceil \text { and } j=1,2 .\end{cases} \\
f\left(y_{(i-1), j}\right) & =2 i, \text { if } i=1,2, \ldots,\left\lfloor\frac{n}{2}\right\rfloor \text { and } j=1,2 . \\
f\left(x_{n-(i-1)} x_{n-i}\right) & =2 i+1, \text { if } i=1,2, \ldots,\left(\left\lfloor\frac{n}{2}\right\rfloor-1\right) . \\
f\left(x_{n-(i-1)} y_{n-(i-1)}\right) & =2 i+j-2, \text { if } i=1,2, \ldots,\left\lfloor\frac{n}{2}\right\rfloor \text { and } j=1,2 . \\
f\left(x_{1} x_{n}\right) & =1 .
\end{aligned}
$$

For $n=3$,

$$
f\left(x_{i} y_{i, j}\right)= \begin{cases}j, & \text { if } i=1 \\ j+1, & \text { if } i=2\end{cases}
$$

For $n>3$ and $n \equiv 0(\bmod 2)$,

$$
f\left(x_{i} y_{i, j}\right)= \begin{cases}j, & \text { if } i=1,2 \text { and } j=1,2 \\ 2 i+j-6, & \text { if } i=3,4, \ldots,\left\lceil\frac{n}{2}\right\rceil \text { and } j=1,2\end{cases}
$$

For $n>3$ and $n \equiv 1(\bmod 2)$,

$$
f\left(x_{i} y_{i, j}\right)= \begin{cases}j, & \text { if } i=1,2 \text { and } j=1,2 \\ 2 i+j-6, & \text { if } i=3,4, \ldots,\left\lfloor\frac{n}{2}\right\rfloor \text { and } j=1,2 \\ 2 i+j-5, & \text { if } i=\left\lceil\frac{n}{2}\right\rceil \text { and } j=1,2\end{cases}
$$

For $n \geq 3$ and $n \equiv 0(\bmod 2)$,

$$
f\left(x_{i} x_{i+1}\right)= \begin{cases}2, & \text { if } i=1, \\ 4, & \text { if } i=2 \text { and } n=4, \\ 2, & \text { if } i=2 \text { and } n \neq 4 \\ 2 i-4, & \text { if } i=3,4, \ldots,\left(\frac{n}{2}-1\right) \text { and } n>4, \\ 2 i-2, & \text { if } i=\frac{n}{2} \text { and } n>4 .\end{cases}
$$


For $n \geq 3$ and $n \equiv 1(\bmod 2)$,

$$
f\left(x_{i} x_{i+1}\right)= \begin{cases}2, & \text { if } i=1, \\ 3, & \text { if } i=2 \text { and } n=3 \\ 2, & \text { if } i=2 \text { and } n \neq 3 \\ 2 i-4, & \text { if } i=3,4, \ldots,\left(\left\lceil\frac{n}{2}\right\rceil-1\right) \text { and } n>3 \\ 2 i-3, & \text { if } i=\left\lceil\frac{n}{2}\right\rceil \text { and } n>3 .\end{cases}
$$

Then, by $f$-labeling we know that label of vertices is even positive integer. From the above formula, we get that the upper bound of $\operatorname{res}\left(C_{n} \odot N_{2}\right)$ same as the lower bound and the weight of edges we get,

For even $n$,

$$
\begin{aligned}
w t\left(x_{i} y_{i, j}\right) & =6 i+j-6, \text { if } i=1,2, \ldots, \frac{n}{2} \text { and } j=1,2 . \\
w t\left(x_{n-(i-1)} y_{n-(i-1), j}\right) & =6 i+j-2, \text { if } i=1,2, \ldots, \frac{n}{2} \text { and } j=1,2 . \\
w t\left(x_{i} x_{i+1}\right) & =6 i-2, \text { if } i=1,2, \ldots, \frac{n}{2} . \\
w t\left(x_{n-(i-1)} x_{n-i}\right) & =6 i+3, \text { if } i=1,2, \ldots,\left(\frac{n}{2}-1\right) . \\
w t\left(x_{1} x_{n}\right) & =3, \text { if } n \geq 3 .
\end{aligned}
$$

For odd $n$,

$$
\begin{aligned}
w t\left(x_{i} y_{i, j}\right) & =\left\{\begin{array}{l}
6 i+j-6, \\
6 i+j-5, \quad \text { if } i=1,2, \ldots,\left(\left\lceil\frac{n}{2}\right\rceil-1\right) \text { and } j=1,2, \\
\left.6 \frac{n}{2}\right\rceil \text { and } j=1,2 .
\end{array}\right. \\
w t\left(x_{n-(i-1)} y_{n-(i-1), j}\right) & =6 i+j-2, \text { if } i=1,2, \ldots,\left\lfloor\frac{n}{2}\right\rfloor \text { and } j=1,2 . \\
w t\left(x_{i} x_{i+1}\right) & = \begin{cases}6 i-2, & \text { if } i=1,2, \ldots,\left(\left\lceil\frac{n}{2}\right\rceil-1\right), \\
6 i-5, & \text { if } i=\left\lceil\frac{n}{2}\right\rceil .\end{cases} \\
w t\left(x_{n-(i-1)} x_{n-i}\right) & =6 i+3, \text { if } i=1,2, \ldots,\left(\left\lfloor\frac{n}{2}\right\rfloor-1\right) . \\
w t\left(x_{1} x_{n}\right) & =3, \text { if } n \geq 3 .
\end{aligned}
$$

By this investigate, we can conclude that every edges has different weight, consequently $f$ is edge irregular reflexive $k$-labeling. So, the proof of $\operatorname{res}\left(C_{n} \odot N_{2}\right)$ is completed.

An illustration of edge irregular reflexive $k$-labeling on $C_{n} \odot N_{2}$ can be seen on Figure 2. The black color is label, red color is weight and blue color is name of vertices. 


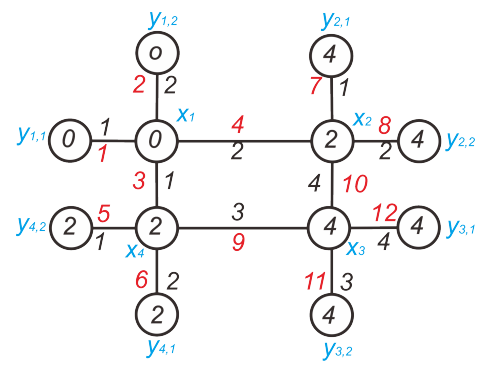

Figure 2. Edge irregular reflexive 4-labeling of $C_{4} \odot N_{2}$.

\section{Concluding remark}

As result the discussion we get conclude $\operatorname{res}\left(\operatorname{sun}_{n}\right)$ or $\operatorname{res}\left(C_{n} \odot N_{1}\right)$ are 3 for $n=3$ and $2\left\lceil\frac{n}{2}\right\rceil$ for $n>3$, while $\operatorname{res}\left(C_{n} \odot N_{2}\right)$ is $2\left\lfloor\frac{n+1}{2}\right\rfloor$ for $n \geq 3$. Moreover, there is open problem for next research about this graph, which still on progress to investigate.

Open problem: What is reflexive edge strength of $C_{n} \odot N_{m}$ for $n \geq 3$ and $m \geq 3$.

\section{Acknowledgement}

This research in this article was supported by RKAT PTNBH Universitas Sebelas Maret fiscal year 2021 through scheme research of grant research group (hgr-uns research) with contract number 260/UN27.22/HK.07.00/2021.

\section{References}

[1] D. Indriati, Widodo, and I. Rosyida, Edge irregular reflexive labeling on corona of path and other graphs. J.P. Conf. Ser., 1489 (2020), 012004.

[2] D. Tanna, J. Ryan, and A. Semaničová-Feňovčíková, Edge irregular reflexive labeling of prisms and wheels, Australas. J. Combin., 69(3) (2017), 394-901.

[3] F. Harary and R. Frucht, On the corona of two graphs, Aequationes Math., 4 (1970), 322-325.

[4] G.K. Dwivedi, Analysis and Desain of Algorithm, Laxmi Publications (P) LTD, New Delhi (2007).

[5] J.A. Galian, A Dynamic survey of graph labeling, Electron. J. Combin., 20 (2017), \#DS6.

[6] J. Ryan, B. Munasinghe, D. Tanna, Reflexive irregular labelings, Unpublished work, 2017.

[7] M. Bača, M. Irfan, J. Ryan, A. Semaničová-Feňovčíková, and D. Tanna, Note on edge irregular reflexive labelings of graphs, AKCE Int. J. Graphs Comb.,16 (2010), 145-157.

[8] M. Bača, S. Jendrol', M. Miller, and J. Ryan, On irregular total labeling, Discrete Math., 307 (2007), 1378-1388. 
[9] R. Boulet, Spectral characterizations of sun graphs and broken sun graphs, Discrete Math. Theor. Comput. Sci., 11:2 (2009), 149-160.

[10] W.D. Wallis and A.M. Marr, Magic Graphs Second edition, Springer Science and Business Media, New York (2013). 